2. A book may be kept four weeks from the date of leaving the library, but the loan may be renewed from time to time by writing to the Society in advance of the day when the book is due.

3. Borrowers should return the books in person or by prepaid express.

\title{
CATALOGUE OF THE LIBRARY.
}

\section{Journals.}

In case the Society has already any bound volumes, these are indicated in the list. Where no bound volumes are indicated, the journal has recently been placed upon the exchange list, and only the current unbound numbers are in the library. The journals are listed alphabetically according to the places of publication.

Amsterdam.

Nieuw Archief voor Wiskunde, Ser. 1, Vols. 1-20; Ser. 2, Vols. 1-6 (1875-1905).

Revue semestrielle des Publications mathématiques, Vols. 1-13

(1893-1905). Tables des Matières, 1892-1902.

Wiskundige Opgaven met de Oplossingen, Vols. 1-8 (1882-1902).

\section{BALTIMORE.}

American Journal of Mathematics, Vols. 1-27 (1878-1905); Index, Vols. 11-20. BerLIN.

Akademie der Wissenschaften, Abhandlungen (selected mathematical memoirs), 1892-94, 1897, 1901, 1903, 1904; Sitzungsberichte, 1857-1868, 1870-1881, 1891-1904; Indexes, 1836-1858, 1859-1873, 1874-1881.

Jahrbuch über die Fortschritte der Mathematik, Vols. 15-33 (1883-1902).

Journal für die reine und angewandte Mathematile, Vols. 96, 97, 109, 125-129 (1884-1905).

Sitzungsberichte der Berliner mathematischen Gesellschaft, Vol. 1. Unterrichtsblätter für Mathematik und Naturwissenschaften, Vols. 9-10 (1903-1904).

Veröffentlichungen d. K. Preus. Geodätischen Instituts, Vols. 1-2, $11,19$.

BOLOGNA.

Il Bolletino di Matematica, Vols. 1-3 (1902-1904).

R. Accademia delle Scienze, Memorie, Ser. 5, Vols. 8-10; Ser. 6, BORDEAUX.

Vol. 1 (1899-1904); Rendiconti, New Series, Vols. 4-8 (1899-1904).

Société des Sciences de Bordeaux, Memoires, Ser. 4, Vols. 1-5; Ser.

5, Vols. 1-5; Ser. 6, Vols. 1-3 (1893-1903) ; Procès-verbaux, 18941904. Boston.

American Academy of Arts and Sciences, Proceedings, Vols. 2640 (1890-1905); Memoirs, Vol. 12 (1902).

T'echnology Quarterly, Vols. 4-17. 
BRAY-SUR-SEINE.

Journals des Géométres-Lxperts, Vols. 1-3, 9-12 (1893-1905).

Brisbane, Queensland.

Proceedings of the Royal Society, Vols. 15-18 (1900-1904).

Brussels.

Annales de la Société Scientifique, Vols. 26-29 (1902-1905);

Index, 1875-1901.

Annuaire astronomique de Observatoire Belgique, 1906.

Annuaire de l'Académie Royale, Vols. 68-71 (1902-1905).

Bulletin de la Classe des Sciences de l'Académie Royale, 1902-1904. Mémoires couronnés et autre Mémoires de l'Académie Royale,

Vols. 60-66 (1900-1904).

Mémoires couronnés et Mémoires des Savants étrangers de

l'Académie Royale, Vols. 58-62 (1899-1904).

BuCHAREST.

Gazeta Matematica, Vols. 1-10 (1895-1904).

BUdAPEST.

See LeIPzIG.

Buenos Aires.

Anales de la Sociedad Cientifica Argentina, Vols. 55-58 (19031904).

Cambridge, Eng.

Cambridge Philosophical Society, Proceedings, Vols. 10-12 (1899-

$1904)$; Transactions, Vols. 17-19 (1899-1904).

Cambridge, Mass.

Annals of Mathematics, Ser. 1, Vols. 1-12; Ser. 2, Vols. 1-6 (1884-1905).

Cherbourg.

Mémoires de la Société Nationale des Sciences naturelles et mathématiques de Cherbourg, Vols. 31-32, 34 (1897-1904).

Chicago.

The Astro-Physical Journal, Vols. 1-22 (1895-1905).

The Monist, Vols. 1-7, 9-15 (1890-1905).

The Open Court, Vols. 11-19 (1897-1905).

School Science and Mathematics, Vols. 3-5 (1903-1905).

School Mathematics, Vol 1.

Christiania.

Archiv for Mathematik og Naturvidenskab, Vols. 21-26 (18991904).

Videnskabs-Selskabet $i$ Christiania, Forhandlinger, 1898-1899, 1901-1904; Skrifter, 1898-1904; Oversigt, 1899-1900.

Città di Castello.

Le Matematiche pure ed applicate, Vols. 1-2 (1901-1903).

CoImbra.

Jornal de Sciencias mathematicas e astronomicas, Vols. 9-15 (1889-1905).

Copenhagen.

Nyt Tidsskrift for Matematik, Ser. A, Vols. 11-16 (1900-1905) ;

Ser. B, Vols. 11-12, 14-15 (1900-1905).

Oversigt d. k. Danske Videnskabernes Selskabs Forhandlinger, 1900-1904. 
Cracow.

Bulletin International de l'Académie des Sciences de Cracovie, 1900-1904.

Katalog, Literatury Naukowej Polskiej, Vols. 2-3.

Des MoInes.

Dublin.

The Analyst, Vol. 10 (1883).

Royal Dublin Society, Scientific Proceedings, New Ser., Vols.

1-10 (1878-1902) ; Scientific Transactions, Ser. 2, Vols. 1-8 (1877-

1903); Index to Proceedings and Transactions, 1877-1898.

EDiNBURGH.

Annals of the Royal Observatory, Vol. 1 (1902).

Proceedings of the Mathematical Society of Edinburgh, Vols.

1, 11-23 (1883-1905) ; Index, Vols. 1-20.

Royal Society of Edinburgh, Proceedings, Vols. 3-4, 10-12, 15-24

(1850-1903) ; Transactions, Vols. 37-39, 42 (1891-1902).

ERLANGEN.

Sitzungsberichte der physicalisch-medicinischen Societät zu Erlangen, Vols. 25-36 (1893-1904).

Fort MonRoE.

Journal of the United States Artillery, Vols. 1-23 (1892-1905).

Ghent.

Mathesis, Ser. 1, Vols. 7-10; Ser. 2, Vols. 1-10; Ser. 3, Vols. 1-4 (1897-1904).

Glasgow.

Proceedings of the Glasgow Philosophical Society, Vol. 30 (1898$1899)$.

GötTingen.

Akademie der Wissenschaften, Nachrichten math.-phys. Klasse, 1892-1905; Geschäftiche Mittheilungen, 1894-1903, 1905.

Grenoble.

Annales de la Faculté des Sciences de l'Université de Grenoble, Vols. 13-16 (1901-1904).

HALIFAX.

Proceedings and Transactions of the Nova Scotia Institute of Sciences, Ser. 2, Vols. 1-2.

Hamburg. See Leipzig.

ITHACA.

Bulletin of the American Physical Society, Vols. 1-2, Vol. 3, No.

1 (last) (1899-1902).

Journal of Physical Chemistry, Vols. 1-9 (1896-1905).

KASAN.

Bulletin de la Société physico-mathématique de Kasan, Vols. $1-5,7-13$ (1891-1903).

KHARKOF.

Communications of the Mathematical Society of Kharkof, Ser. 2, Vols. 5-7 (1897-1902).

KIEFF.

University Isvistija, Vols. 43-44 (1903-1904).

LAWRENCE.

Kansas University Quarterly, Ser. A, Vols. 9-10 (1900-1901).

Kansas University Science Bulletin, Vols. 1-2 (1902-1905). 
LEGHORN.

Periodico di Matematica, Ser. 1, Vols. 2-11; Ser. 2, Vols. 12-18; Ser. 3, Vols. 19-20 (1888-1905).

LEIPZIG.

Supplemento al Periodico di Matematica, Vols. 2-8 (1898-1904).

Archiv der Mathematik und Physik, Ser. 3, Vols. 1-9 (1901$1905)$.

Bibliotheca Mathematica, 1887-1903.

Jahresbericht der Deutschen Mathematiker-Vereinigung, Vols.

1-9; New Ser., Vols. 11-14 (2 sets, except Vols. 1-4) (1890-1905). Mathematische Annalen, Vols. 41-43, 45-60 (1893-1905).

Mathematische und naturwissenschaftliche Berichte aus Ungarn, Vols. 1-20 (1882-1902).

Mittheilungen der mathematischen Gesellschaft in Hamburg, Vols.

2-3 (1890-1900).

Zeitschrift für Mathematik und Physik, Vols. 44-52 (1899-1905).

Sächsische Gesellschaft der Wissenschaften, math.-phys. Classe;

Abhandlungen, Vols. 25-28 (1899-1902); Berichte, Vols. 45-56

(1893-1904).

Zeitschrift für mathematischen und naturwissenschaftlichen Unterricht, Vols. 23-29 (1892-1898).

\section{LEMBERG.}

Sammelschrift der mathematisch-naturwissenschaftlichen Section

der Sevcenko-Gesellschaft der Wissenschaften, Vols. 9-10 (1903$1904)$.

Lick ObServatory, MT. Hamilton.

Lick Observatory Contributions, Vols 3-5 (1893-1895); Publications, Vols. 1-6 (1887-1903).

LIEGE.

Mémoires de la Société Royale des Sciences de Liége, Ser. 2, Vols. 19-20; Ser. 3, Vols. 1-5 (1897-1904).

LiMA.

Revista de Ciencias, Vols. 1-7 (1898-1904).

Livorno. See Legirorn.

LoNDoN.

Educational Times, 1897-1904.

Journal of the Institute of Actuaries, Vols. 35-38 (1901-1904).

Royal Society, Philosophical Transactions, Ser. A, Vols. 192-204

(1899-1905) ; Proceedings, Vols. 62-76 (1898-1905); Year Book,

Vols. 1-2 (1896-1898).

Proceedings of the London Mathematical Society, Vols. 1-35

(1865-1903) ; Ser. 2, Vols. 1-3 (1903-1905) ; Index, Vols. 1-30.

List of Members, 1865-1899.

Mathematical Gazette, Vols. 1-2 (1896-1904).

Messenger of Mathematics, Vols. 6- $7,24-34$ (1894-1905).

Quarterly Journal of Pure and Applied Mathematics, Vols. 31-36 (1899-1905).

LUXEMBURG.

Institut Grand-Ducal, Publications (Section des Sciences naturelles et mathématiques), Vols. 8-9, 15, 17-21, 23-27 (1865-1904). MADRID.

Archivo de matematicas, 1896-1897 (last).

Memorias de la Real Academia de Ciencias, Vols. 14, 20 (1890, 1903 ).

Rivista de la Real Academia de Ciencias, Vols. 1-2 (1904-1905). 
MANCHESTER.

Memoirs and Proceedings of the Manchester Literary Sociely, Vols. 37-49 (1892-1904).

Marseilles.

Annales de la Faculté des Sciences de Marseilles, Vols. 11-13 (1901-1903).

Mexico.

Memorias y Rivista de la Sociedad Cientifica Antonio Alzate, Vols. 2-20 (1888-1903).

MILAN.

Annali di Matematica pure ed applicata, Ser. 3, Vols. 2-11 (1899$1905)$; General Index, 1850-1897.

Reale Istituto Lombardo di Scienze e Lettere, Rendiconti, Ser. 2, Vols. 33-37 (1900-1904); Memorie, Vol. 19 (1900).

MONTPELLIER.

Mémoires de l'Académie des Sciences de Montpellier, Ser. 2, Vols. 1-2 (1893-1900).

Moscow.

Transactions of the Mathematical Society of Moscow, Vols. 1-24

(1866-1903) ; Index, Vols. 1-15.

Physico-Mathematical Gazette, Vols. 1, 4-11, 13-22 (1880-1904).

MUNICH.

Hochschul-Nachrichten, Vol. 13 (1902-1903).

NAPLES.

Accademia delle Scienze fisiche e matematiche di Napoli, Atti, Ser. 2, Vols. 8-11 (1899-1902); Rendiconti, Vols. 34-43 (1895$1903)$.

NEW YoRK.

Annual Register of the American Mathematical Society, 18911905.

Bulletin of the American Mathematical Society, Vols. 1-11 (18941905 ) ; General Index, 1891-1904; Transactions, Vols. 1-6 (1900$1905)$ (2 sets).

Bulletin of the Association of Teachers of Mathematics in the Middle States and Maryland, Vols. 1-2 (1904-1905).

Bulletin of the New York Mathematical Society, Vols, 1-3 (18911894) (2 sets).

Papers and Transactions of the Actuarial Society of America. Vols. 1-8 (1889-1904).

Physical Review, Vols. 16-21 (1903-1905).

Science, New Ser., Vols. 1-22 (1895-1905).

NORTHFIELD.

Astronomy and Astrophysics, 1892-94.

Popular Astronomy, Vols. 10-13 (1902-1905).

Odessa.

Viestnile Opytnoy Fisiki, Vols. 24-25.

Society of Naturalists of New Russia, Mathematical Section, Zapiski, Vol. 20 (1902).

Palermo.

Atti dell' Accademia di Scienze di Palermo, Ser. 3, Vols. 1-6 (1891-1901).

Il Pitagora, Vols. 2-10 (1896-1904). 
Rendiconti del Circolo matematico di Palermo, Vols. 1-19 (18841905 ) ; Annuario, 1898, 1900, 1904.

Paris.

Bulletin des Sciences mathématiques et physiques élémentaires, Vols. 1-10 (1895-1904).

Bulletin de la Société mathématique de France, Vols. 21-33 (18931905 ).

Bulletin de la Société philomatique de Paris, New Ser., Vols. 4-6

(190]-1904).

Bulletin des Sciences mathématiques, Vols. 27-28 (1903).

Bureau des Longitudes, Annuaire, 1903-1906.

Comptes rendus de l'Académie des Sciences de Paris, Vols.

136-139 (1895-1904).

Journal de Mathématiques pures et appliquées, Ser. 5, Vols. 1-10

(1895-1905).

L'Education mathématique, Vols. 5-6 (1902-1903).

L'Enseignement mathématique, Vols. 4-6 (1902-1904).

L'Intermediare des mathématiciens, Vols. 1-11 (1894-1904).

Nouvelles Annales de Mathématiques, Ser. 1, Vols. 1-20 (1842$1861)$; Ser. 2, Vols. 1-11 (1862-1872), 13-19 (1874-1879) ; Ser. 4, Vols. 3-4 (1903-1904).

Revue de l'Institut catholique, Vols. 7-9 (1902-1904).

Revue générale des Sciences, Vols. 13-16 (1902-1905).

Revue de Mathématiques spéciales, Vols. 13-14 (1902-1904).

Pavia.

Rivista di Fisica, Matematica e Scienze Naturali, Vols. 7-12 (1903-1905).

\section{Philladelphia.}

American Philosophical Society, Proceedings, Vols. 29-43 (1891$1904)$; Transactions, Vol. 20 (1902); List of Surviving Members, 1892.

PISA.

Annali della Scuola normale superiore, Vols. 1-9 (1871-1904).

Prague.

Casopis pro pestováni mathematiky a fysiky (Journal of Mathematics and physics ), Vols. 21, 23-25, 27-33 (1892-1903) ; Index to Vols. 1-30.

Jednota českych Mathematikú Sbornik, Vols. 1-9. (See Books other than Journais, under Prague.

Rozpravy Ceské Akademie Césaře Františka Josefa. (The memoirs relating to mathematics only.) Vols. 1-12 (1891-1903).

Věstnik Královské Ceské Spolècnosti Náuk (Proceedings of the Bohemian Academy of Sciences ), 1899-1902; Bulletin Internationale, Vols. 7-9.

\section{RALEIGH.}

Journal of the Elisha Mitchell Scientific Society, Vols. 9-12 $(1892-1895)$.

\section{RENNES.}

Travaux Scientifiques de l'Université, Vols. 1-3 (1902-1905).

ROME.

Accademia dei Lincei, Atti, Vol. 4; Rendiconti, Ser. 5, Vols. 1-7, 9-14 (1892-1905).

Pontificia Accademia dei Nuovi Lincei, Atti, Vols. 48-57 (1894$1903)$; Memorie, Vols. 15-18 (1898-1901). 
Societa Italiana delle Scienze, Memorie di Matematica e di Fisica, Vols. 12-13 (1902).

Rivista di Artiglieria e Genio, Vols. 1-4, New Ser., Vols. 1-3.

San Francisco.

Publications of the Astronomical Society of the Pacific, Vols. 1-16 (1889-1904).

Springfield, Mo.

The American Mathematical Monthly, Vols. 1-12 (1894-1905).

S'T. Lours.

Transactions of the St. Louis Academy of Sciences, Vols. 5-14 (1886-1904).

St. Petersburg.

Bulletin de l'Académie des Sciences, Vols. 1, 5-32 (1860-1888); Ser. 5, Vols. 1-16 (1890-1903).

STockhoum.

Acta Mathematica, Vols. 1-29 (1882-1904).

\section{STUTTGART.}

Mathematisch-naturwissenschaftliche Mitteilungen, Ser. 1, Vols.

1-5 (1884-1892) ; Ser. 2, Vols. 1-6 (1899-1904).

Zeitschrift für Vermessungswesen, Vols. 31-34 (1902-1905).

Tokyo.

Sugaku-Butsurigaku Kuai Kiji (Journal of the Physico-Mathematical Society of Tokyo), Vols. 1-5, 7-8; Ser. 2, Vol. 1.

Toulouse.

Annales de la Faculté des Sciences de l'Université de Toulouse, Ser. 1, Vols. 6-12; Ser. 2, Vols. 1-6 (1891-1904).

Bulletin de l'Académie des Sciences de Toulouse, Vol. 3 (18991900).

Mémoires de l'Académie des Sciences de Toulouse, Ser. 10, Vols. 1-4 (1901-1904).

Turin.

Reale Accademia di Scienze di Torino, Atti, Vols. 30-40 (1895-

1905; Index, Vols. 21-30; Memorie, Ser. 2, Vols. 48-55 (1899-1905).

Bulletino di Bibliografia e Storia delle Scienze matematiche, Vols. 5-7 (1902-1904).

Formulaire de Mathématiques, Vols. 1-4 (1895-1902).

Revue de Mathématiques, Vols. 1-7 (1891-1901).

ULM.

Jahresheft des Vereins für Mathematik und Naturvissenshaften, 1903.

UPSALA.

VIENNA.

Universitets Arsskrift, 1901-1904.

Monatshefte für Mathematik und Physik, Vols. 1-16 (1890-1905). Sitzungsberichte der kaiserlichen Akademie der Wissenschaften in Wien, math.-naturwiss. Classe, Vols. 108-113 (1899-1904).

\section{WARSAW.}

Travaux de la Société de Naturalists, Imp. Université de War. saw, Vols. 1-3 (1891-1895).

Prace matematyezno-fizyczne, Vols. 1-16 (1888-1905).

Tablice Matematyczno-fizyczne, 1904.

Wiadomosci matematyczne, 1897-1904. 
WASHINGTON.

Publications of the U. S. Naval Observatory, Ser. 2, Vols. 1-5 (1900-1905).

Smithsonian Institution, Reports, 1885-87, 1889-90, 1895-1904.

Zaragossa.

Revista trimestral de Matematicas, Vols. 1-4 (1901-1904). ZURICH.

Vierteljahrschrift der naturforschenden Gesellschaft, Vols. 34-49 (1889-1904).

II. Books other than Journals. Accessions in 1905.

Ahrens, W. Mathematische Unterhaltungen und Spiele, Leipzig, 1901.

BaIllard, B. et Bourate, H. Correspondence d'Hermite et de Stieltjes, Vols. 1 and 2, Paris, 1905.

Belfast. Queen's College Calendar, 1905-6.

BolyaI, W. und Gauss, C. F. Briefwechsel, Leipzig, 1899.

BoLzA, O. Lectures on the calculus of variations, Chicago, 1904.

Boole, M. E. Preparation of the child for science, Oxford, 1904.

BoRel, E. Leçons sur les séries divergentes, Paris, 1901.

Leçons sur les séries a termes positifs, Paris, 1902.

Bourget, H. See Baillard, B.

BRISTOL. University College Calendar, 1905-6.

CARDIFF. Calendar of the University College of South Wales, 1904-5.

Clay, C. M. Examples in algebra, New York, 1905.

Clement-Jones, A. Notes on analytical geometry, Oxford, 1903.

Copenhugen. D. Kgl. Danske Vidensk. Selsk. Skrifter. (Mathematical parts only, including memoirs by the following authors: BERNoulli, J., Brahes, T., Broager, P. D., Bronsted, J. N., Buchwaldt, F., Cagnoli, Colding, D'Arrest, H. I., Degen, C. F., Gram, J. P., Hansen, P. A., Hansen, P. C. V., Hertzsprung, S., Juel, C., Jurgensen, C., Lande, Jorenz, L., Olufsen, C. F. R., Örsted, H. C., Ramus, C., Schjellerup, H. C. F. C., Schmidten, H. G., Steen, A., Thiele, T. N., Valentiner, H., Wessel, C., Zeuthen, H. G., 1784-1899.)

Couturat, L. L'algèbre de la logique, Paris, 1905.

Cunningham, A. J. C. Binary canon, London, 1900.

Czuber, E. Wahrscheinlichkeitsrechnung, Parts 1 and 2, 1902.

DUeLIN. Examination papers of the Royal University, 1904.

Calendar of the Royal University, 1905.

Duhem, P. Recherches sur l'hydrodynamique, Paris, 1904.

ENCYCLOPÄDIE der mathematischen Wissenschaften, Vol. 1, Leipzig, 1904.

Estanave, E. Essai sur la sommation de quelques séries trigonométriques, Paris, 1903.

Foering and Lambert. Plane and spherical trigonometry, 1905.

Gauss, C. F. Werke, Vol. 9, 1903.

See Bolyai, W.

Goursat, E. Cours d'analyse mathématique, Vol. 2, Paris, 1905.

Goursat-Hederick. Mathematical analysis, Vol. 1, Boston, 1905. 
Granvilis, W. A. Elements of the differential and integral calculus, Boston, 1904.

HaLsted, G. B. Rational geometry, New York, 1904.

Hammer, E. Sechstellige Tafel der Werte von $\log \frac{1+x}{1-x}$, Leipzig, 1902.

Hedrick, E. R. See Goursat.

Hensed, K. and Landsberg, G. Theorie der algebraischen Funktionen einer Variablen und ihre Anwendungen auf algebraischen Kurven und Abelsche Integrale, Leipzig, 1902.

Hermite, Chr., Poincare, H., Rouche, E. Oeuvres de Laguerre, Vol. 2, Paris, 1905.

See PiCARD.

HILL, G. W. Collected mathematical works, 1905.

Holzm ̈̈́ler, G. Ingenieur-Mathematik, Das Potential, Leipzig, 1898.

HubBell, H. The geometry of Euclid, 1861.

Hunson, R. W. H. T. Kummer's quartic surface.

Kiepert, I., Stegraman, M. Grundriss der Differential- und IntegralRechnung, Paris, Hanover, 1900-1901.

KüBıER, B. J. Woher kommen die Weltgesetze?

LAMBert. See Foering.

Landsberg, G. See Hensel.

LARMor, J. Aether and matter, Cambridge, 1900.

LAURENT, H. Sur le principes fondamentaux de la théorie des nombres.

Lechalas, G. Introduction à la géométrie générale, Paris, 1904.

LeVAvasseur, R. Groupes d'ordres finis et groupes finis continus, Lyons, 1904.

Lindei.̈̈r, E. Calcul des residus, Paris, 1904.

MCMurry, C. A. Special method in arithmetic, New York, 1905.

Osgood, W. F. Collected papers, Vols. I, II.

Pascal, E. Lezioni di calculo infinitesimale, Part I, integrale; Part II, differenziale, Milan, 1902-1903.

Picard, E. Oeuvres de Charles Hermite, Vol. 1, Paris, 1905.

Sur le develloppement de l'analyse, Paris, 1905.

Traité d'analyse, Paris, 1896.

Poincare, H. Electricité et optique, la lumière et les théories électrodynamiques, 1901 .

See Hermite, CH.

Prague. Jednota českých mathematiku sbornik.

Vol. 1. Weyr, E. Projectivná geometrie, 1898.

Vol. 2. KoLÁČ́̌, F. Hydrodynamika, 1899.

Vol. 3. STUdNičKa, F. J. Úvod do nauky o determinantech, 1900.

Vol. 4. Strouhal, C. Mechanika, 1901.

Vol. 5. Weyr, E. Počet differenciâlný, 1902.

Vol. 6. Strouhal, C. Akustika, 1903.

Vol. 7. Stunnrcka, F. J. Analytické geometrie, V rovině, 1903.

Vol. 8. Koloušsek, J. Mathematická theorie dủchodi̊ jistých a pujcek annuitních, 1904.

Vol. 9. KoLÁčK, F. Electrina a magnetismus, 1905. 
Riemann, B.-Staht, H. Elliptische Functionen, Leipzig, 1899.

Rouche, E. See Hermite, Ch.

SchöNFELd, G. A. Die Kromme van den vierden Graad in de vierdimensionale Ruimte, Groningen, 1904.

SmITH, D. E. Portfolio of portraits of eminent mathematicians, Chicago, 1905.

Solvert, M. Mémoires sur une classe de fonctions elliptiques, Brussels, 1903.

Stahl, H. See Riemann, B.

Stegemann, M. See Kisepert, L.

Stringham, I. Uniplanar algebra, San Francisco, 1893.

SYDNEY.. Calendar of the University, 1905.

Van Vleck, E. B., White, H. S., Woods, F. S. The Boston Colloquium Lectures on Mathematics, New York, 1905.

WANGERIN, A. Verhandlungen der Breslauer Naturforscher-Versammlung, Leipzig, 1905.

White, H. S. See Van Vleck, E. B.

Wilson, J. C. On the Traversing of Geometrical Figures, Oxford, 1905.

Woods, F. S. See Van Vleck, E. B. 